\title{
Design of a Model to Create the Web Services using Service-Oriented Development
}

\author{
Preeti Gupta \\ Department of Computer \\ Science \& Engineering \\ ASET, Amity University \\ Sector-125, Noida
}

\author{
Abhishek Singhal \\ Department of Computer \\ Science \& Engineering \\ ASET, Amity University \\ Sector-125, Noida
}

\author{
Varun Rajpoot \\ Salesforce Developer \\ (Cloud Platform) \\ Astrea IT Pvt. Ltd. \\ Sec-64, Noida
}

\begin{abstract}
Today, large numbers of web services are available which puts impacts in the service-oriented development. The emerging growth in service-oriented architecture (SOA) leads users to access wide range of web services which define proper standards. Through this paper, an approach is defined for creating the web services in the environment of serviceoriented development. Proper model is designed to discuss about the three phase of developer phase, broker phase \& client phase. These phases are further divided into sub phases. The development process is initiated by describing the each phases in the model. The functionalities and the requirements are discussed thoroughly. This paper gives the approach to develop the web services which focus on the different parameters and enhancing the scope for better services availability.
\end{abstract}

\section{Keywords}

Interoperability, process, reusability, services, serviceoriented development, service repository, service-oriented architecture (SOA)

\section{INTRODUCTION}

The emerging growth of technology has brought much improvement in the field of Service-Oriented Architecture. SOA is an architectural approach whose focus is to enhance the reusability and increase the productivity. The main requirement is to provide the services which tend to be highly cohesive and loosely coupled. Reducing the development cost, time and other resources by constructing a logical architecture provides a single service connectivity platform [1].

Services used to have the features of more and more use of reusable components, dynamically composed to be strong cohesion, increase the interoperability for the new and existing models etc. Service-oriented architecture deals with the functional and non- functional requirements. Main objective concerned in this paper is to propose the methodology which designs the model for creating and reusing the web services. Many implementations techniques based on the different approaches have been proposed earlier. But still lacking in properly categorizing and identifying the different entities [2].

Components and services together hold the appropriate integration that meets the requirement. In different models, developers face the situation which is aligned with business approach in terms of the requirements.

Study of different information system gives the adequate results in quality, agility and also the success rate. Development of the complex services for better changes in terms of usage and cost [3].
In this paper, section 2 discussed about some related work. Section 3 defines the motivation, reason behind describing the model. In section 4 proposed a model which describes the different phrases in three broad categories to reveal the associated needs and fulfilling the needs. Last section discussed about the conclusion and Future related scope.

This paper includes the different requirements for designing the three phases which focus on different perspective of Developers, Broker and Client:

Evaluation and analysis of various requirements for the services oriented development.

Study and analysis various existing methodology to create web service.

Achieving interdependency, reusability in web services using the Component Based Service Engineering.

Identify relevance of propose techniques to create web service model.

Promoting the reusability leads to a new shift in the serviceoriented environment. The demands of client are increasing which leads to high cost value in designing and implementing the web services. Web services variability is described for better reusability in different scenarios by dividing the process into the sub processes. Different views are discussed in terms of services and business process puts attention towards the functional and non- functional requirements. By focusing on the Variable representation is used for improving the reusability in the web services. Requirements are further categorized in terms of similarities and dissimilarities about the applications services. Missing of a comprehensive approach in the services is founded which supports the variability at the different phases of life cycle development process [4].

\section{RELATED WORK}

All Compound Based Software Engineering is a new paradigm from the traditional software architecture to facilitate the software development more effective and economically sound by ensuring the reuse components. Component Based Software Development ensures to give more throughputs in terms of light weighted model and reusability. Today web services are reused to construct applications on the web, providing with the different services by maintaining the transparency with respect to the multiple environments and technologies. So focus to maintain the new view transparency which leads to service-oriented software development. In the Service-Oriented, there is benefit with respect to the firewalls friendly behavior [5]. 
Usage of web services realized the importance of interoperability. Benefits of using the combined approach model driven and service-oriented architecture are together used to develop the interfaces for the application system at the abstraction level describes the web services applications. Approach is built for the existing application usage which is applied for the development of web interface. The existing applications are integrated to provide the better advanced functionality for a user to access [6].

\section{MOTIVATION}

Services based software implementation is a advanced paradigm to move new and advanced technologies. Several existing approaches have been proposed, to focus the Software Development Life Cycle [5].

The main focus is to enhance the services on the web. Therefore it requires a complete new model which should consider the service-oriented process.

\section{PROPOSED METHODOLOGY}

Our contribution focus on designing a model which focuses on the different research objectives like functional and nonfunctional requirements. The three phrases defined below is been categorized.

\subsection{Developer Phase:}

\section{i. Gathering Information \& Requirements}

First phase is Gathering Information \& Requirements in which information is gathered from the different entities. Different role entities are involved like client, developer, management, actual user.

Sometimes the situation arises when the requirements of actual user is neglected. Requirements are different in every perspective. So, focusing on these measures, all perspectives are been considered for gathering the more useful and appropriate information.

ii. Analysis

In the next phase of analysis, there is a role of developer and analysis. Analyze the different functional and non- functional requirements. Approach of analysis the situation and environment which is functional and non- functional both. Analysis the existing web services, looking forward to its strong and weak points and work accordingly.

Analysis Reusability is divided into three categories. First category talks about the variation study. In variation study, discussed about the changes or variations of services is observed.

Second category is talks about the view code. That is view code dynamically.

Last category is talks about the Reusability. Code reusability, the code which is already developed can be reuse again. Different Components developed in the different languages can be accessed which increases the interoperability.

\section{iii. Design}

Moving to the next phase is designing. Designing the services according to the requirements which talks about the graphical user interface which is more attractive and ease to use. Design the services accordingly so that it saves the resources, also makes the service lighter and use the less space.

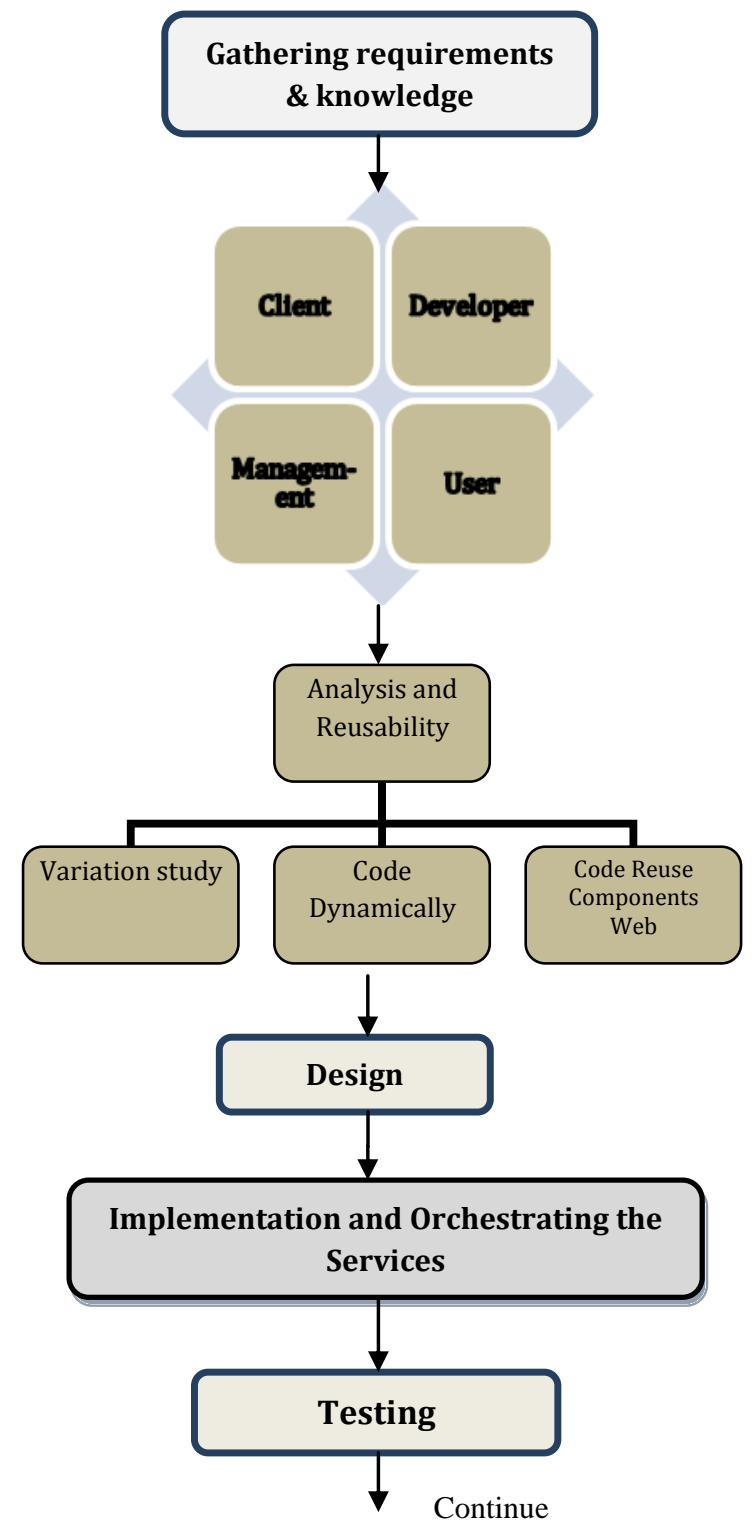

Fig. 1(a) Developer Phase

iv. Implementation and Integration Services

Considering the implementation and integration of services phase in which developing the services. Using the different environment services is built. Select the services which are observed more beneficial and optimal to use. Also keeping in the mind about the more reusability, reliability. Integrating the different web services will lead to high cohesion and low coupling interdependency.

v. Testing

Services are ready to perform testing. Test the services to avail with the more scalability in the services. During the developer phase, test the services in the developer point of view and the functionality defined in the documentations. 


\subsection{Broker Phase}

Second major role play is of Broker who is actually makes the services available for clients to access showing in the fig.1(b). Developer develops the services and broker asks the services to publish in the repository.

i. Service Repository

In the service repository, all the services are available to use and also, the new services is added into it. Here the role of service discovery where the existence services been chosen up to access it, instead of implementing the same services again and again just call the exist service.

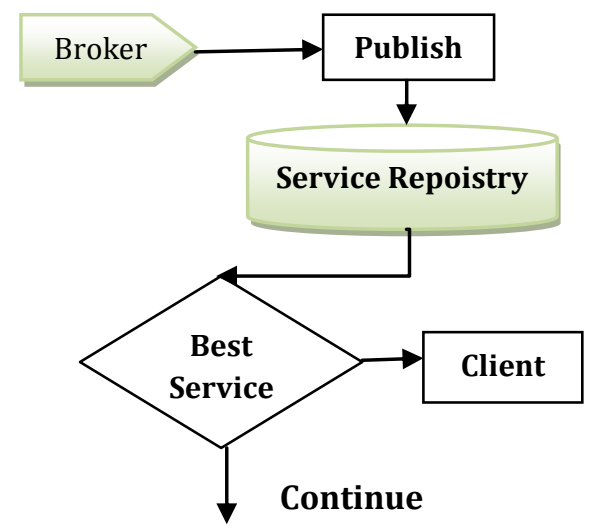

Fig. 1(b) Broker Phase

Focus on the service discovery which is more suitable reliable and also impactful is been observed and access it.

Discover the mechanism which gives the better result in orchestrating the resources and knowledge more appropriately.

\subsection{Client Phase}

The third phase define is Client phase in which the focus is on the client and the actual user defined in the Fig.1(c).

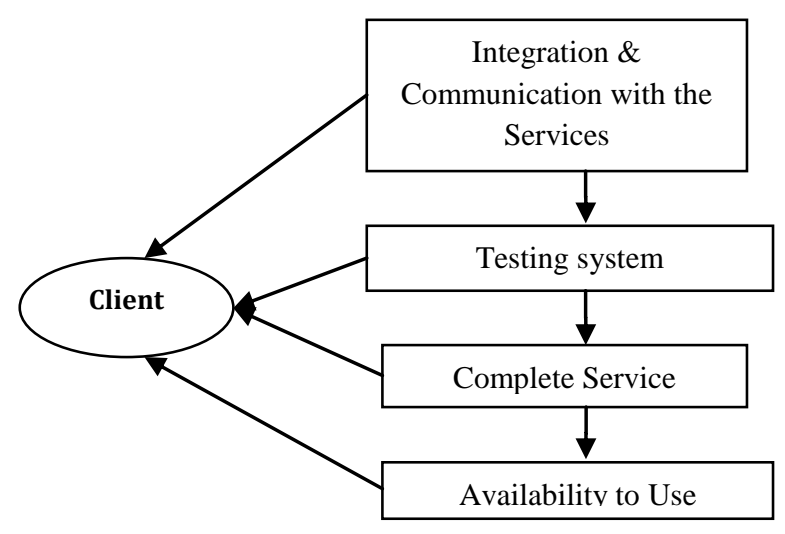

Fig. 1(c) Client Phase

\section{i. Best Service}

The client chooses the best services according to its requirements which can be ease to use and adds the required functionality in it. ii. Service Orchestration

Integrate the services together to make it available at the same environment for the user.

Now perform the testing according to the user environment. Run the web services to test it accordingly. The complete system is now available to use.

\section{CONCLUSION}

Requirement in the service-oriented environment puts special impact in the services on the web. The focus of requirement engineering in service-oriented is not on how to build the system but rather what appropriate model we wish to build. We observed the different paradigms for the developing the services. Emphasis more on the different perspectives so that the essential needs does not get unfocussed. Performing the testing on both the ends of the developer and client will provide better compatibility and interoperability. This model can be applied for implementing individual services. As well as integrating the different services running on different platforms altogether. For the future work should be related with the more additional levels in details.

\section{REFERENCES}

[1] Ashish Seth, Himanshu Agarwal, Ashim Raj Singla "Designing a SOA based model". pp. 1-7. In proceeding of 2011 ACM SIGSOFT Software Engineering Notes archive Volume 36.

[2] Hongming Cai, Fenglin $\mathrm{Bu}$ and Lihong Jiang "A busines-driven methodology for service-oriented information system development”. pp. 292-299. In Proceeding of Parallel and Distributed Processing Symposium Workshops \& PhD Forum (IPDPSW), Shanghai . 26th International IEEE, 2012.

[3] Galster, M. Univ. of Calgary, Calgary, AB Bucherer, E. "A Taxonomy for Identifying and Specifying NonFunctional Requirements in Service-Oriented Development". pp. 345 - 352 In proceeding of Services Part I, 2008. IEEE Congress in Honolulu, HI. 10.1109/SERVICES-1.2008.51.

[4] Fazziki, A.E. Fac. des Sci., Semlalia Univ. Cadi Ayyad, Marrakech, Morocco Lakhrissi, H. ; Yetognon, K. ; Sadgal, M, "A Service Oriented Information System: A Model Driven Approach". pp. 466-473. In proceeding of 2012 Eighth International Conference in Naples, Signal Image Technology and Internet Based Systems (SITIS).

[5] Chakir, B. AlQualsadi team, Md V Souissi Univ., Rabat, Morocco Fredj, M.; Nassar, M. "Promoting reuse in web services by managing variability". pp. 712 - 717. In proceeding of 2012 International Conference Multimedia Computing and Systems (ICMCS), Tangier. IEEE, 10.1109/ICMCS.2012.6320274.

[6] Mardiana , Keijiro Araki , Yoichi Omori "MDA and SOA Approach to Development of Web Application Interface”. pp. 226-231. In proceeding of 2011 Conference TENCON. IEEE, 2011.

[7] Marzieh Emadi, Reza Asgari Moghadam, Mohamad Davarpanah Jazi, Faranak Bahredar "An Improved methodology for Service Oriented Architecture". pp. 350-354. International Conference of Computer Science and Automation Engineering (CSAE), Zhangjiajie, China. IEEE, 2012. 10.1109/CSAE.2012.6272790 
[8] Maurizio Leotta, Gianna Reggio, Filippo Ricca, Egidio Astesiano "Towards a Lightweight Model Driven Method for Developing SOA Systems Using Existing Assets". pp. 51-60. In proceeding of 2012 14th IEEE International Symposium on Web Systems Evolution (WSE), Trento, Italy. IEEE, 2012.

[9] Youcef Baghdadi "A framework to select an approach for web services and SOA development". pp. 277-282. IEEE 2012 International Conference on Innovations in Information Technology (IIT), Abu Dhabi. 10.1109/INNOVATIONS.2012.6207747.

[10] Wangping Xiong, Xianbo Xiao , Ji -cheng Shu and XianZhou "Research on Service Oriented Architecturebased data mining system". pp. 844-846. In proceeding of 2012 International Conference of Computer Science and Information Processing (CSIP). IEEE, 10.1109/CSIP.2012.6308985

[11] Hendrik Moens, Eddy Truyen, Stefan Walraven, Wouter Joosen, Bart Dhoedt and Filip De Turck "Developing and Managing Customizable Software as a Service Using Feature Model Conversion". In proceeding of Network Operations and Management Symposium (NOMS). pp. 1295-1302. IEEE, 2012.
[12] Jawaria Sadiq, Athar Mohsin and Fahim Arif "Quantifying Non-functional Requirements in Service Oriented Development" pp. 224-229. In proceeding of Frontiers of Information Technology (FIT), Islamabad. IEEE 2011, 10.1109/FIT.2011.48.

[13] Wagner Arnaut , Kathia Oliveira and fernanda Lima "OWL-SOA: A Service Oriented Architecture Ontology Useful During Development Time And Independent From Implementation Technology". pp. 523-532. Fourth International Conference of Research Challenges in Information Science (RCIS), Nice, France. IEEE 2010.

[14] Mahdi Fahmideh, Mohsen Sharifi, Pooyan Jamshidi, Fereidoon Shams, Hassan Haghighi "Process Patterns for Service-Oriented Software Development". pp. 1-9. 2011 Fifth International Conference Research Challenges in Information Science (RCIS), Gosier. 2011.

[15] Fayçal, H. Univ. of Sci. \& Technol. Houari Boumedienne, Algiers, Algeria Habiba, D.; Hakima, M. "Integrating legacy systems in a SOA using an agent based approach for information system agility". Pp. 338 - 343. IEEE 2010 International Conference Machine and Web Intelligence (ICMWI) in Algiers. 